\title{
Prediction of thioguanine-induced cytotoxicity by dual-parameter flow cytometric analysis
}

\author{
Jonathan Maybaum ${ }^{1 *}$, Paul Ting ${ }^{1}$, and Clare E. Rogers ${ }^{2}$ \\ Departments of 'Pharmacology and ${ }^{2}$ Internal Medicine, University of Michigan Medical School, Ann Arbor, Michigan
}

Summary. A method is presented for the quantitative analysis of delayed cytokinetic effects resulting from the treatment of L1210 cells with 6-thioguanine (TG). By using dual-parameter (DNA/protein) flow cytometry, we could observe the accumulation of late $\mathrm{S} / \mathrm{G} 2 / \mathrm{M}$ cells with abnormally high green fluorescence (i.e., protein content), indicative of unbalanced growth. The use of mitotic cells from a pseudotetraploid line (HT29) as external markers for both red and green fluorescence facilitated highly reproducible measurement of the mean green fluorescence $\left(\mathrm{GFL}_{\text {mean }}\right)$ of the arrested late $\mathrm{S} / \mathrm{G} 2 / \mathrm{M}$ population. We found that the dose dependence of the observed $\mathrm{GFL}_{\text {mean }}$ values followed the same unusual biphasic pattern as did cytotoxicity in this cell line, indicating that this parameter might be a suitable means of predicting TG-induced toxicity in vivo. We propose that the low background expected for this kind of measurement would make it particularly appropriate for the analysis of clinical specimens (e.g., mononuclear bone marrow cells) from leukemic patients receiving thiopurines, to monitor (and, hopefully, predict) their response to treatment.

\section{Introduction}

The thiopurines 6-thioguanine (TG) and 6-mercaptopurine (MP) are used in combination with other drugs for the treatment of various leukemias. An unusual feature of these agents is that they can produce a "paradoxical" doseresponse curve for cytotoxicity. That is, cell kill increases with drug concentration only up to a given point, beyond which more drug actually produces less cell kill (Table 1) $[3,6,7]$. It has been proposed that this phenomenon is due to an interaction between the two most prominent biochemical effects of thiopurine treatment, namely, purine starvation and analog incorporation into DNA.

We have previously reported that experiments using single-parameter (1P) flow cytometric analysis (of cellular DNA content) are consistent with this proposal [7]. These studies suggested that the range of drug concentrations

\footnotetext{
* Recipient of a Faculty Development Award from the Pharmaceutical Manufacturers Association Foundation.

Offprint requests to: J. Maybaum, 4302A Upjohn Center, University of Michigan Medical School, Ann Arbor, MI 48109-0504, USA
}

that produced the greatest degree of cell kill also gave rise to the most pronounced accumulation of cells with an apparent late S/G2 complement of DNA, characteristic of permanent cell-cycle arrest due to thiopurine incorporation into DNA under these circumstances [5, 6]. Furthermore, high drug concentrations that induced submaximal cell kill appeared to cause less late S/G2 arrest.

Because of the irregular character of DNA content distributions derived from these drug-perturbed populations, we could not quantitate these effects satisfactorily in a single-parameter system. However, since it had previously been shown that cells experiencing thiopurine-induced, delayed late $\mathrm{S} / \mathrm{G} 2$ arrest also appear to undergo unbalanced growth [2], we hypothesized that dual-parameter (2P) analysis of DNA and protein content of TG-treated populations might permit a simple, quantitative analysis of the extent of this arrest without requiring the use of a mathematical model of the population. This paper describes such an analysis using L1210 cells in culture and reports the quantitative correlation between TG-induced cytotoxicity and accumulation of late $\mathrm{S} / \mathrm{G} 2$ cells with substantially elevated protein content. In addition, the implications of these results for the use of thiopurines in humans is discussed.

\section{Materials and methods}

Cell growth and sample preparation. L1210 cells were routinely grown as suspension cultures in RPMI 1640 medium containing $10 \%$ horse serum at $37^{\circ} \mathrm{C}$ in a humidified $5 \%$

Table 1. Biphasic dose-response curve for the loss of clonogenicity in L1210 cells treated with TG."

\begin{tabular}{lll}
$\begin{array}{l}\text { TG concentration } \\
(\mu M)\end{array}$ & $\begin{array}{l}\text { Surviving } \\
\text { fraction }\end{array}$ & $\begin{array}{l}\text { Cell kill } \\
(-\log \text { surviving fraction })\end{array}$ \\
\hline 0 & 1.00 & 0 \\
0.008 & 0.951 & 0.022 \\
0.04 & 0.148 & 0.830 \\
0.2 & 0.0026 & 2.59 \\
1.0 & 0.0156 & 1.81 \\
5.0 & 0.0180 & 1.74 \\
25.0 & 0.0283 & 1.55 \\
\hline
\end{tabular}

a Cells were exposed for $12 \mathrm{~h}$, washed free of drug, then plated in soft agar. These data have been presented elsewhere in graphical form [7] 


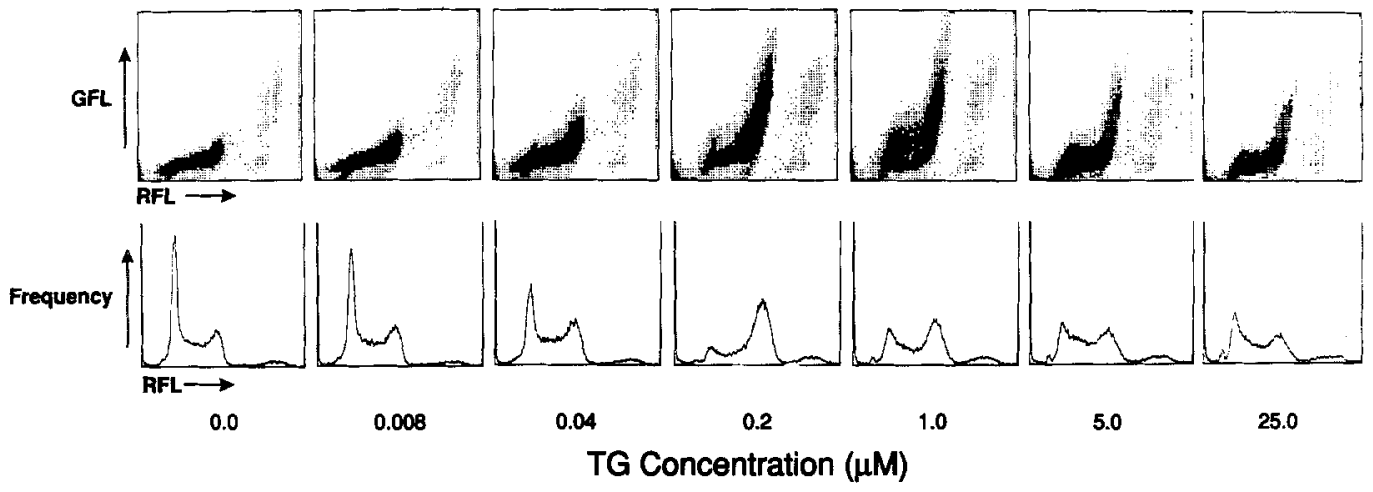

Fig. 1. Dose-dependent cytokinetic effects of TG treatment in L1210 cells. Cells were treated for $12 \mathrm{~h}$, washed free of drug, incubated $24 \mathrm{~h}$ in fresh medium, then fixed. The top panels show 2-parameter histograms (X-axis $=\mathrm{RFL}$; Y-axis $=\mathrm{GFL}$ ) of cells analyzed after staining for DNA (propidium iodide) and protein (FITC) content. The bottom panels show the corresponding single-parameter RFL histograms

$\mathrm{CO}_{2}$ atmosphere. HT29 cells, which were used as markers, were grown as monolayer cultures in McCoy's 5 A medium containing $10 \%$ fetal bovine serum. Mitotic HT29 cells were obtained by treating newly confluent monolayers with $0.2 \mu \mathrm{g} / \mathrm{ml}$ colcemid (Sigma Chem. Co., St. Louis, Mo) for $16 \mathrm{~h}$, at which time the flasks were agitated and the detached mitotic cells decanted, washed with Hank's buffered saline solution (HBSS, GIBCO, Grand Island, $\mathrm{NY}$ ), and fixed with 70\% EtOH to a final concentration of $50 \% \mathrm{EtOH}$.

For drug treatments, TG (Sigma) was dissolved in a small volume of $0.1 \mathrm{~N} \mathrm{NaOH}$, filter-sterilized, and then diluted in medium to the appropriate concentration. Cells were exposed to TG for $12 \mathrm{~h}$ (one doubling time), after which they were washed twice with fresh medium and then incubated for an additional $24 \mathrm{~h}$ in normal medium. This protocol enables the expression of delayed late $\mathrm{S} / \mathrm{G} 2$ arrest, which is characteristic of TG treatment $[1,4,9]$. Cells were fixed in ethanol as described above and stored at $4^{\circ} \mathrm{C}$ until the day before analysis, at which time they were stained essentially as previously described [8]. One million fixed L1210 cells were combined with $2.5 \times 10^{5}$ fixed Trout red blood cells (TRBCs) and $2.5 \times 10^{5}$ fixed HT29 cells, washed twice with HBSS, then resuspended in $0.05 \mu \mathrm{g} / \mathrm{ml}$ fluorescein isothiocyanate (FITC; Sigma) and incubated at room temperature for $2 \mathrm{~h}$. Cells were then pelleted and resuspended in $1 \mathrm{ml}$ solution containing $18 \mu \mathrm{g} / \mathrm{ml}$ propidium iodide (PI) in HBSS and stored overnight at $4^{\circ} \mathrm{C}$.

Flow cytometry. A Coulter EPICS C was used for these analyses, operating at an excitation wavelength of $488 \mathrm{~nm}$ and using a 514-nm-band pass filter for linear green fluorescence (GFL) and a 600-nm-long pass filter for linear red fluorescence (RFL). The algorithm used to prepare the instrument for these studies was as follows.

Four histograms were used: (1) H1: 2P RFL $\times$ GFL, $64 \times 64$ channels, gated from a window in $\mathrm{H} 4$; (2) $\mathrm{H} 2$ : 1P GFL gated from a bitmap in $\mathrm{H} 1$; (3) H3: 1P RFL, gated from a window in $\mathrm{H} 4$; and (4) $\mathrm{H} 4$ : $1 \mathrm{P} \log$ forward angle light scatter (LFLS).

$\mathrm{H} 2, \mathrm{H} 3$, and $\mathrm{H} 4$ were each comprised of 256 channels. Control cells and one or two drug-treated samples were preliminarily run to establish initial gating and amplification parameters. Cursors were first adjusted in H4 (LFLS) to exclude debris and clumps of cells. Next, the photo- multiplier (PMT) voltage for RFL was adjusted to place the peak of the TRBC markers in channel $32 / 256$ of histogram H3. A bitmap was then drawn around the HT29 marker population in $\mathrm{H} 1$, which was used to gate for $\mathrm{H} 2$. The purpose of this step was to enable the determination of the mean GFL value for the main HT29 population while excluding a minor subpopulation of this bimodal distribution. The PMT voltage for GFL was then adjusted such as to place the mean GFL of $\mathrm{H} 2$ into channel $120 \pm 2$ (which was equivalent to channel 30 in H1). Data were then collected for each sample until 40,000 events were registered in histogram 1.

\section{Results}

Figure 1 shows a series of $2 \mathrm{P}$ RFL $\times$ GFL histograms and corresponding 1P RFL histograms obtained from a single, typical experiment. With respect to the effect of TG treatment on cell-cycle distribution under these conditions, these samples appear to show the same dose-dependent effect on late $\mathrm{S} / \mathrm{G} 2 / \mathrm{M}$ accumulation we previously reported [7]. In addition, there also appears to be a substantial increase in the green fluorescence of the late S/G2/M-arrested cells, consistent with unbalanced growth, as had been predicted. Since one of our main goals was to establish a practical means of quantitating these effects, we defined the following scheme for analysis of this type of experiment.

The Cytologic software package (Coulter Electronics) was used for the determination of the numbers of cells in various parts of $2 \mathrm{P}$ histograms. This software enables the operator to use two horizontal and two vertical cursors to define a bounded area, after which the number of cells within this area, as well as the mean RFL and GFL values of the cells therein, are reported. As shown in Fig. $2 \mathrm{~A}$, the first operation was to quantitate the total number of L1210 cells collected. This was accomplished by setting the lefthand vertical cursor (low $\mathrm{X}$ value) to channel 8 (i.e., the peak TRBC channel) and the right-hand vertical cursor (high $\mathrm{X}$ value) to a position between the L1210 cell population and the marker HT29 population. To maintain consistency, we defined the position of the high X cursor to be $75 \%$ of the way between the low X cursor and the center of the HT29 population. The lower horizontal cursor (low Y value) was set to channel 1 and the upper horizontal cursor 
A

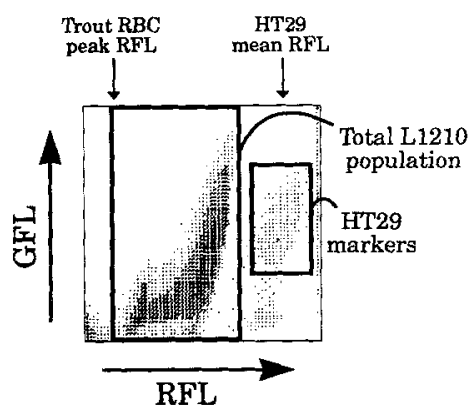

B

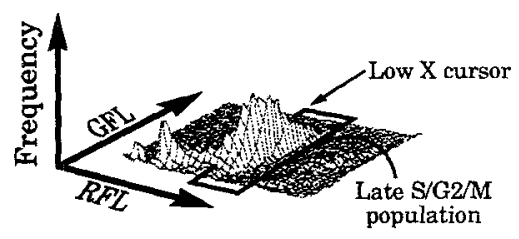

C

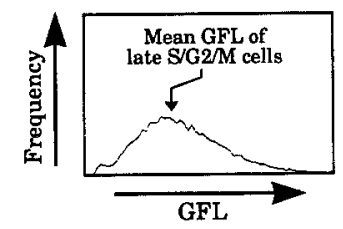

Fig. 2. Algorithm for setting cursors for quantitative analysis. A. Two points of reference were defined on the X-axis, the peak TRBC channel (obtained from the 1P RFL histogram) and the channel of mean RFL for the HT29 markers, as determined by Stat Pack. The right-hand boundary of the L1210 population was placed at the channel $75 \%$ of the way between these two reference points; the left-hand boundary was set at the TRBC peak channel. Low $Y$ and high Y cursors were set at channels 1 and 63 , respectively. B. The region containing late $\mathrm{S} / \mathrm{G} 2 / \mathrm{M}$ cells was delineated by setting the low X cursor to the channel $35 \%$ of the way between the reference points described above. C. A IP projection was made to show the distribution of GFL within the late $S / G 2 / M$ cell population, and the channel number representing the mean value of this population is denoted by the arrow

(high Y value) was set to channel 63, which included any particles whose GFL values were off-scale in the positive direction. The number of cells within the bounded area under these conditions was defined as the total L1210 cell population detected.

The second step (Fig. 2B) was to delineate the total late $\mathrm{S} / \mathrm{G} 2 / \mathrm{M}$ population observed. The key decision to be made here concerned the placement of the low $\mathrm{X}$ cursor, dividing late $\mathrm{S} / \mathrm{G} 2 / \mathrm{M}$ cells from the rest of the $\mathrm{L} 1210$ population. Our strategy was to place this cursor as high as possible without impinging on the cell population with elevated GFL. After examination of all of the $2 \mathrm{P}$ histograms, we designated the low $X$ position to be $35 \%$ of the way between channel 8 and the center of the HT29 population. Although this placement is empirical, the results of these studies were relatively insensitive to the exact positioning of this cursor (see below). Finally, the GFL values for the population bounded in Fig. 2B (i.e., the late $\mathrm{S} / \mathrm{G} 2 / \mathrm{M}$ cells) were projected onto a single-parameter histogram (Fig. 2C) from which the mean GFL of these cells was determined.

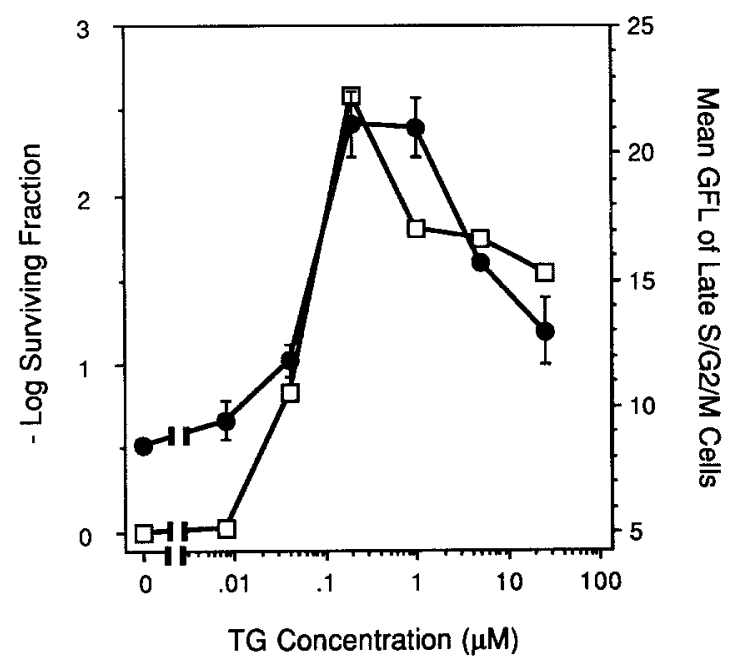

Fig. 3. Relationship between the mean GFL of late $\mathrm{S} / \mathrm{G} 2 / \mathrm{M}$ cells and TG-induced cytotoxicity. Values $\square$ for -log surviving fraction (from Table 1) are plotted with for mean GFL values ( \pm SE) from Table 2

Table 2. Quantitative analysis of the cytokinetic effects of TG treatment in L1210 cells ${ }^{a}$

\begin{tabular}{lllll}
\hline $\begin{array}{l}\text { TG con- } \\
\text { centration } \\
(\mu M)\end{array}$ & $\begin{array}{l}\text { Mean GFL of } \\
\text { late S/G2/M cells } \\
\text { (channel number } \\
\pm \mathrm{SE} \text { ) }\end{array}$ & $\begin{array}{l}\text { Change in mean GFL } \\
\text { resulting from repositioning } \\
\text { of low X cursor }\end{array}$ \\
\cline { 3 - 4 } & & +2 channels & -2 channels \\
\hline 0 & $8.47 \pm 0.18(2.1 \%)$ & $3.42 \%$ & $4.01 \%$ \\
0.008 & $9.45 \pm 0.76(8.0 \%)$ & $3.60 \%$ & $4.02 \%$ \\
0.04 & $11.79 \pm 0.64(5.4 \%)$ & $3.14 \%$ & $5.42 \%$ \\
0.2 & $21.11 \pm 1.28(6.1 \%)$ & $2.37 \%$ & $1.99 \%$ \\
1.0 & $21.02 \pm 1.19(5.7 \%)$ & $3.46 \%$ & $3.09 \%$ \\
5.0 & $15.70 \pm 0.08(0.5 \%)$ & $3.95 \%$ & $4.39 \%$ \\
25.0 & $12.98 \pm 1.30(10.0 \%)$ & $3.47 \%$ & $4.62 \%$ \\
\hline
\end{tabular}

a $\mathbf{L} 1210$ cells were exposed to drug for $12 \mathrm{~h}$, then washed free of drug, incubated for $24 \mathrm{~h}$ in normal medium, and fixed in ethanol. The extent of unbalanced growth in this population, as indicated by a disproportionately high protein content in late $\mathrm{S} / \mathrm{G} 2 / \mathrm{M}$ cells, was measured as shown in Fig. 2 and is expressed as the mean green fluorescence (GFL) of this subpopulation. The averaged values from three independent experiments are presented in column 2. These values were also recalculated after the low X cursor had been moved either 2 channels higher or 2 channels lower than its original position to simulate misplacement of this cursor. The changes in mean GFL that resulted from this repositioning are given in columns 3 and 4

The above analysis was carried out using data from three separate experiments in which cells were treated with a range of TG concentrations, and the resulting values for the mean GFL of late $\mathrm{S} / \mathrm{G} 2 / \mathrm{M}$ cells were calculated (Table 2) and plotted along with previously determined values for $\log$ cell kill induced by such treatments. Figure 3 demonstrates that the observed increase in GFL of late $\mathrm{S} / \mathrm{G} 2 / \mathrm{M}$ cells follows the same bimodal pattern as the cytotoxicity curve and that the maximum for each parameter occurs within the same range of drug concentrations, $0.2-1 \mu M$.

As mentioned earlier, the algorithm for the selection of a channel number for setting the low $\mathrm{X}$ cursor to divide 
the L1210 cell population between apparent late $\mathrm{S} / \mathrm{G} 2 / \mathrm{M}$ DNA cells and G1/early S cells was designed empirically, based on the position of the L1210 population with increased GFL. To determine the sensitivity of this analytical procedure to the placement of the low $\mathrm{X}$ cursor, we recalculated the values for the mean GFL of late S/G2/M cells after the low X cursor had been reset either two channels higher or two channels lower than its original position. The results of this study (Table 2) show that with one exception, movement of the low X cursor by two channels in either direction resulted in a distortion of the mean GFL value of $<5 \%$. Since the magnitude of this potential distortion was much lower than that of the changes in GFL resulting from cytotoxic treatments, we conclude that the precision of the scheme used for setting the low $\mathrm{X}$ cursor is sufficient to avoid artifactual changes in mean GFL due to a variable definition of the late $\mathrm{S} / \mathrm{G} 2 / \mathrm{M}$ population.

\section{Discussion}

Like most antimetabolites, TG induces multiple biochemical lesions whose relative importance may vary, depending on factors including treatment schedule, drug concentration, and the type of cells used. Previous results have indicated that the conditions favoring the delayed accumulation of cells in late $\mathrm{S} / \mathrm{G} 2 / \mathrm{M}$ are correlated with maximal cell kill in vitro [7]. One of the long-term objectives of the present study is to determine if the effectiveness of this drug as an antileukemic agent in humans is related to the extent of such arrest. For this objective to be reached, a practical means must be available for the quantitation of the degree of this arrest in clinical specimens.

One problem associated with the use of $1 \mathrm{P}$ histograms for the analysis of changes in cell-cycle distribution is that drug-treated populations often exhibit irregular DNA distributions. Since cell-cycle analysis algorithms are usually based on the assumption that G1 and G2 peaks will be Gaussian and symmetrical, the irregular 1P RFL distributions seen following TG treatment are difficult to evaluate quantitatively. The decision to examine DNA and protein content in a dual-parameter system was predicated on the supposition that cell death following late $\mathrm{S} / \mathrm{G} 2 / \mathrm{M}$ arrest is due to unbalanced growth, i.e., continued RNA and protein synthesis in the absence of DNA synthesis [2]. On this basis, we predicted that the delayed late $\mathrm{S} / \mathrm{G} 2 / \mathrm{M}$ arrest caused by TG would be accompanied by the appearance of cells with inordinately high cellular protein levels, as was indeed found (Fig. 1).

At least two criteria must be met for the $2 \mathrm{P}$ DNA/protein analysis described here to be useful as proposed in the clinical setting. First, the assay must give quantitatively reproducible results. As shown in Table 2, the standard error associated with the determination of the mean GFL for late $\mathrm{S} / \mathrm{G} 2 / \mathrm{M}$ cells was $\leq 10 \%$ of the average value in all cases. It should be noted that the use of HT29 marker cells for adjusting the machine prior to data collection was a crucial factor in obtaining this degree of precision. Although the RFL photomultiplier voltage was usually not changed between samples, the GFL photomultiplier voltage quite frequently required modification for the placement of the marker cells in a given location. This suggests that the FITC staining procedure was somewhat variable in its efficiency.

Second, the parameter derived from this analysis must reflect cell kill. Most cytotoxic drug treatments elicit nu- merous biochemical effects on cells, which increase with the drug concentration, eventually reaching a plateau value. In the case of TG, however, the biphasic dose-response pattern for cytotoxicity requires that any putative parameter for predicting cell kill must also exhibit such a pattern, with a maximal value in the appropriate concentration range. As shown in Fig. 3, the mean GFL for late S/G2/M cells meets this requirement.

Another potential virtue of quantitating late $\mathrm{S} / \mathrm{G} 2 / \mathrm{M}$ cells with high GFL as an in vivo index of drug response is that the background value for this parameter is negligible, enabling the detection of small effects in response to drug treatment. In contrast, measurement of drug-induced changes in the number of cells in any particular cell-cycle phase using RFL alone would require the subtraction of some baseline value.

In summary, we found that L1210 cells experiencing delayed late $\mathrm{S} / \mathrm{G} 2 / \mathrm{M}$ arrest as a consequence of TG treatment exhibited a coordinate rise in cellular protein content. We presented a procedure enabling the objective quantitation of this effect, the application of which revealed that the extent of the accumulation of cells with high GFL values correlated with the loss of clonogenicity in the experimental system used here. We intend to apply this method as a means of analyzing the effects of TG on leukemic cells in humans receiving this drug.

Acknowledgements. We are grateful to the Children's Leukemia Foundation of Michigan for support, to Dr. Jerry Hudson for helpful discussions and advice, and to Mr. David Shelton for technical assistance.

\section{References}

1. Barranco SC, Humphrey RM (1971) The effects of beta-2'-deoxythioguanosine on survival and progression in mammalian cells. Cancer Res 31:583

2. Horakova K, Navarova J, Paterson ARP (1974) The delayed cytotoxic effect of 6-mercaptopurine. Characterization of the unbalanced growth in HeLa cells produced by 6-mercaptopurine. Biochim Biophys Acta 366: 333

3. Matsumura S, Hoshino T, Weizsaecker M, Deen DF (1983) Paradoxical behavior of 6-mercaptopurine as a cytotoxic agent: decreasing cell kill with increasing drug dose. Cancer Treat Rep 67: 475

4. Maybaum J, Mandel HG (1981) Differential chromatid damage induced by 6-thioguanine in CHO cells. Exp Cell Res 135: 465

5. Maybaum J, Mandel HG (1983) Unilateral chromatid damage: a new basis for 6-thioguanine cytotoxicity. Cancer Res 43: 3852

6. Maybaum J, Hink LA, Roethel WM, Mandel HG (1985) Dissimilar actions of 6-mercaptopurine and 6-thioguanine in Chinese hamster ovary cells. Biochem Pharmacol 34: 3677

7. Maybaum J, Morgans CW, Hink LA (1987) Comparison of in vivo and in vitro effects of continuous exposure of L1210 cells to 6-thioguanine. Cancer Res 47: 3083

8. Pollack A, Moulis H, Block NL, Irvin GL III (1984) Quantitation of cell kinetic responses using simultaneous flow cytometric measurements of DNA and nuclear protein. Cytometry 5: 473

9. Wotring LL, Roti Roti JL (1980) Thioguanine-induced S and G2 blocks and their significance to the mechanism of cytotoxicity. Cancer Res 40: 1458

Received 27 September 1988/Accepted 7 February 1989 